\title{
Le doute et la douleur
}

Initiations et affects en pays sénoufo (Côte d'Ivoire)

Doubt and pain: initiations and affects among the Senufo (Ivory Coast)

\section{Marianne Lemaire}

\section{(2) OpenEdition}

\section{Journals}

Édition électronique

URL : http://journals.openedition.org/span/810

DOI : 10.4000/span.810

ISSN : 2268-1558

Éditeur

École pratique des hautes études. Sciences humaines

Édition imprimée

Date de publication : 1 décembre 2008

Pagination : 193-218

ISSN : 0294-7080

Référence électronique

Marianne Lemaire, «Le doute et la douleur », Systèmes de pensée en Afrique noire [En ligne], 18| 2008, mis en ligne le 05 juin 2013, consulté le 01 mai 2019. URL : http://journals.openedition.org/span/810 ; DOI : $10.4000 /$ span. 810 


\title{
Le doute et la douleur \\ Initiations et affects en pays sénoufo (Côte d'Ivoire)
}

\author{
Marianne Lemaire* \\ Chargée de recherche au CNRS \\ Centre d'études des mondes africains (CEMAf) \\ CNRS / Université Paris I/EPHE / \\ Université de Provence
}

La souffrance endurée par les impétrants au cours de leur initiation a de longue date retenu toute l'attention des anthropologues. Mais l'importante littérature anthropologique qui lui est consacrée s'est le plus souvent intéressée à la souffrance physique infligée à un collectif de jeunes garçons dans le cadre de l'initiation obligatoire qui leur donne accès au statut d'adulte de plein droit ${ }^{1}$. Dans le contexte sénoufo, il paraît toutefois intéressant de mettre en parallèle la douleur des impétrants de l'initiation obligatoire et masculine appelée por $\boldsymbol{0}$ et la souffrance endurée par certaines femmes dans le cadre de l'initiation élective au sandogi. Prolongeant une réflexion antérieure sur la souffrance telle que les Sénoufo la valorisent ${ }^{2}$ - une souffrance plénière, nécessairement composée d'une double dimension physique et morale, et qui, en tant que telle, peut être surmontée -, cet article se propose d'examiner la distribution des souffrances physique et morale entre les deux principales initiations sénoufo, pour l'une obligatoire et masculine, pour l'autre élective et féminine. Quand effectivement les impétrants masculins du pro expérimentent une souffrance principalement physique, les impétrantes de l'initiation au sandogi, elles, endurent une souffrance principalement morale, à travers le sentiment de doute qui les tenaille tout au long de leur initiation, et aussi bien avant de la subir et après l'avoir achevée. L'examen de ce sentiment et des raisons pour lesquelles il est l'objet d'un aussi méticuleux entre-

\footnotetext{
* Je remercie

Dominique Casajus,

Dorothée Dussy,

Michael Houseman et

Eric Jolly pour leurs précieuses suggestions à propos de ce texte.
${ }^{1}$ L'initiation au poro fait partie des initiations habituellement appelées «tribales». L'adjectif ne me paraissant pas satis. faisant, je parlerai ici d'initiations obligatoires donnant accès au statut d'adulte de plein droit.
2 Lemaire, 2005.

Éprouver l'initiation

Systèmes de pensée en Afrique noire, 18, 2008, pp. 193-218 
tien pourrait permettre de porter un regard nouveau sur la distinction entre initiations obligatoires donnant accès au statut d'adulte de plein droit et initiations électives, ainsi que sur celle entre initiations masculine et féminine.

\section{La souffrance physique des impétrants du pors}

Le pro des Sénoufo Tyebara, autrefois composé de trois phases de sept ans chacune, est aujourd'hui restreint à une phase unique, le tyologi, au cours de laquelle les novices répondent au nom de tyolobele ( $\mathrm{pl}$. de tyolowi). Obligatoire, l'initiation au pro conduit tout homme à intégrer, avec sa promotion, un "bois sacré ", autrement dit une association secrète composée des membres masculins de quelques matrilignages alliés. Elle lui permet par là de parachever son identité de Sénoufo Tyebara, mais aussi de s'assurer une «bonne mort " grâce à la participation des initiés du pro aux rituels qui succèderont à son décès. Une telle assurance a un prix : elle s'acquiert après que le novice a rempli sept ans durant, outre de nombreuses prestations matérielles, d'aussi nombreuses prestations rituelles auxquelles les Tyebara donnent le nom de "travail». Leurs prestations rituelles sont en effet "le travail des tyolobele", qui, en les accomplissant, accomplissent " le travail de leur mère " ou " le travail de la vieille femme du village ». " Mère ", "vieille femme du village ", "chef du village ", "femme jalouse ", "femme rancunière ": autant d'appellations différentes pour référer à un même être initiatique, Katyeleqwi, dont les Sénoufo situent la résidence dans le bois sacré, où il met tous les sept ans de nouveaux enfants au monde, et d'où il ne sort que pour enterrer l'un d'eux ${ }^{3}$.

$\mathrm{Du}$ " travail ", dont le paradigme en pays sénoufo est le travail agricole, les prestations rituelles des impétrants du pro comportent en effet cette dimension essentielle qu'est la souffrance physique. L'initiation au pro est éprouvante : si elle contraint les novices à donner des biens matériels, elle les contraint surtout à donner d'eux-mêmes à travers l'effort et les forces que requièrent les travaux qui leur incombent. Sept ans durant, le novice est celui qui cultive 
les champs de ses aînés le jour, et qui, la nuit, "balaye les routes " conduisant aux villages souvent éloignés où il est chargé de transmettre un message ou d'accomplir des prestations funéraires. Ces travaux initiatiques font de lui un être en perpétuel mouvement, condamné à mobiliser toutes ses ressources physiques tout le temps que dure son initiation.

Plus éprouvants encore sont cependant les travaux que, durant les sept premiers jours de leur initiation, les novices accomplissent dans le bois sacré où ils sont reclus. Nus et démunis, ils pénètrent dans le "village " de Katyeleqwi par une froide nuit sans lune de la saison sèche. Là, la « vieille femme " ne tarde pas à les atteler à des travaux qui se révèlent être de véritables épreuves. Courir autour du bois sacré sous une pluie de cailloux, plonger la tête, le temps jugé nécessaire par l'initiateur, dans un canari d'eau pimentée $e^{4}$ autant de tâches pénibles que Katyelęwi leur ordonne successivement de réaliser avant que de les mettre symboliquement à mort et de les remettre non moins symboliquement au monde. Ce sont ces travaux que, le jour du rituel kafowi qui clôt leur septennat initiatique et leur fait atteindre le statut de kafokunbele ("ceux qui ont coupé le kafowi»), les jeunes initiés évoquent dans leurs chants. Par crainte de trop en dire sur leur initiation, la plupart des initiés se contentent d'exprimer leur joie d'être enfin " sortis " du bois sacré et d'avoir obtenu la queue de bœuf et la cendre blanche qui l'attestent :

Je me suis lavé, je me suis lavé pour sortir, je suis sorti avec un crédit

La connaissance que j'ai recherchée est sortie, dansons

Les vieux m'ont donné une queue de bœuf, dansons
Les vieux m'ont donné de la cendre blanche, dansons

Mais d'autres kafokunbele, ceux qui, dit-on, ont le plus douloureusement vécu les travaux initiatiques, n'hésitent pas à exprimer, dans un long chant ininterrompu, les souffrances encore vives qu'ils leur ont causées. Savamment rendu obscur au non-initié ${ }^{5}$, ce chant lui laisse entendre l'essentiel : ô combien éprouvants sont " les travaux de la vieille femme du village ». D’ores et déjà éprouvante est ainsi la
${ }^{4}$ Concernant les épreuves traversées par les novices dans le bois sacré, voir G. Bochet (1993) et A. Zempléni (1993 et 1996).

${ }^{5}$ Si les kafokunbele n'emploient dans leurs chants que des termes courants, ils les emploient en leur attribuant un tout autre sens que le sens courant. 
${ }^{6}$ Le kusuluwi est un aîné des novices qui les guide tout au long de leur parcours initiatique.

${ }^{7}$ Zempléni, 1996 : 37. piste " touffue " qui conduit au "village de quelqu'un " où de tels travaux sont accomplis :

Quand je me suis approché, j'ai vu une grande route et j'ai vu une piste touffue

Et j'ai dit : «Quelle route je vais prendre pour arriver jusqu'au village de quelqu'un? "

Et le kusuluwi ${ }^{6}$ m'a dit: "Toi idiot, toi novice ignorant, regarde la piste touffue,

elle mène au village de quelqu'un. "

Et j'ai emprunté celle-ci comme il fallait l'emprunter.

Qui emprunte cette piste "touffue " plutôt que la route dégagée qui s'offre à lui doit être conscient qu'elle mène à des travaux eux-mêmes "touffus " - à des travaux ardus et pénibles :

Et je suis aussitôt parti leur dire que nous avions terminé les travaux touffus du bois sacré.

Mais c'est un ignorant qui, parce qu'il a traversé une épreuve sans y voir un " travail ", croit les avoir toutes traversées et avoir achevé son initiation :

[...] Et j'ai obtenu cela sans travail

Et j'ai dit : "J'en ai fini avec le travail, je vais maintenant me promener un peu partout puis me coucher dans le village de quelqu'un et y dormir profondément. "

Alors qu'il y a aussi la panthère dans la forêt [...].

Car si les travaux initiatiques sont aussi pénibles, c'est qu'ils sont innombrables. Ainsi que le souligne Andras Zempléni, ils n'attestent rien de moins qu'une "vision abyssale du labeur indéfini de la connaissance [...] au principe de l'ethos et de l'éthique d'endurance des Sénoufo ${ }^{7}$ ":

Et j'ai couru dire au kusuluwi que j'en avais fini avec les choses de l'initiation

Alors que les affaires de l'initiation sont plus nombreuses que les graines de fonio

Quand tu en as fini avec l'une, une autre surgit et s'accroupit à sa place.

Et les chants de fin d'initiation d'évoquer la pénible quête des " os de l'âne " ou des « oreilles d'éléphant " nécessaires aux novices pour compléter leurs « bagages d'homme dur ", ainsi que l'épreuve du "balancement du singe vert ", celle de la "pluie du ciel sans nuage", ou encore celle du 
"chien poilu qui lape " dont les impétrants aimeraient autant qu'il les morde. Arrive cependant un moment où les novices ne sauraient être davantage malmenés, car il n'est pas de brin de paille sans nœud pour enrayer la progression de la braise qui le dévore : paille.

C'est pourquoi je dis qu'il y a un nœud sur tout brin de

À l'issue de ces épreuves nocturnes, le groupe des novices offrent ce triste spectacle: "Le rideau tombe à l'aube lorsque les néophytes, réduits à l'état de cadavre, la tête pendant en arrière, sont introduits, un à un, dans son petit village [celui de la "vieille femme"], d'où ils ne pourront sortir que par une ouverture spéciale ou un trou étroit, le trou de la mangouste, qui représenterait le vagin de leur mère ${ }^{8}$."

Aux côtés de la souffrance physique, inextricablement liée à elle, la souffrance morale n'est pas non plus absente de l'expérience initiatique des novices. Les kafokunbele se remémorent dans leurs chants le sentiment de désarroi que, face aux travaux du bois sacré, ils ont à maintes reprises ressenti :

Et j’ai dit : « Où vais-je trouver un petit chasseur pour les [le phacochère et le chien] tuer et les faire sortir des sillons de nos mères, pour que je puisse régler les autres choses du bois sacré comme il faut les régler?"

Les anciens novices ne font pas l'aveu de leur détresse sans faire celui de leur effroi :

Je me suis arrêté brusquement et je tremblais dans le village de quelqu'un

Mon bagage du bois sacré s'est détaché et est tombé à terre

Mon arc d'homme viril s'est détaché et est lui aussi tombé à terre.

Mener à bien son parcours initiatique réclame donc de mobiliser, outre ses ressources physiques, des qualités qui se situent "sur son cœur" :

Si tu veux prendre la direction du village de quelqu'un,

dépose le cœur de ton enfance dans les vieilles maisons, et prends le cœur de ta maturité pour chercher le village de quelqu'un.

8 Zempléni, 1993 : 7. 
Il s'agit en effet pour le novice de puiser au plus profond de lui-même la détermination qui va lui permettre de surmonter les épreuves auxquelles il est soumis :

\footnotetext{
Ne prends pas le sommeil pour chercher la place du bois sacré comme il faut la chercher

Mets tes mains dans l'eau froide et appuie-les sur ton cœur.
}

Ainsi que Gilbert Bochet l'a relevé, les initiés mettent volontiers en avant, ailleurs que dans leurs chants, la composante morale de leur souffrance : "De l'aveu des intéressés, les épreuves purement physiques leur sont moins pénibles que ces moments d'abandon où ils sont laissés après qu'une savante mise en scène les a contraints de remettre en question leur optique traditionnelle et a créé un certain désarroi ${ }^{9}$. " Mais cette dimension de souffrance morale a beau être essentielle et mériter d'être réévaluée, elle n'est malgré tout pas celle qui est donnée au premier chef à voir aux non-initiés, et n'atteint pas dans l'initiation au pro la même mesure que dans l'initiation au sandogi.

\section{La souffrance morale des futures initiées au sandogi}

Le terme sandogi désigne en premier lieu une puissance dont l'autel consiste en une poterie retournée et enfouie dans la terre. Constitutive de tout matrilignage, cette puissance a pour attribution principale de circonscrire avec précision les limites du groupe exogame, en prohibant les relations sexuelles entre ses membres et en exerçant sur les autres un contrôle sévère. C'est ainsi qu'une amende dite yapeqre, "chose pour balayer ", doit être versée par les partenaires des femmes du matrilignage pour permettre à son aîné de « balayer » la souillure qu'une relation sexuelle y introduit. Faute de quoi la puissance sandogi est susceptible de se manifester en infligeant malheurs et maladies au sein du lignage.

Mais la puissance sandogi peut aussi tourmenter le lignage parce qu'elle veut voir l'un de ses membres intégrer l'institution initiatique elle-même appelée sandogi. Cette 
institution très majoritairement composée de femmes obéit en effet au fonctionnement suivant ${ }^{10}$ : chaque fois qu'une initiée au sandogi (une sandowi) meurt, elle libère dans son matrilignage une place qui ne saurait rester vacante durant un temps trop long. Après qu'un devin a déterminé l'identité et le mobile de la puissance à l'origine des infortunes d'un lignage, il lui revient, lorsque cette puissance est le sandogi, de déterminer l'identité de la personne que la défunte souhaite voir entrer à sa place dans l'institution initiatique.

Contrairement à l'institution initiatique du pro en effet, l'institution sandogi recrute ses membres par voie d'élection : une sandowi défunte choisit la petite fille, la jeune femme ou la femme adulte qu'elle souhaite voir "porter" son sandogi après elle. Ce n'est cependant pas là le seul mode d'élection. Il peut arriver, quoique beaucoup plus rarement, qu'un génie émette le même souhait. D'ailleurs, la succession dans laquelle chaque sandowi vient prendre place après avoir été élue par une défunte s'origine toujours dans une telle élection par un génie : un jour, une ancêtre élue par un génie et ayant porté son sandogi, a, une fois morte, demandé qu'un membre de son matrilignage le "porte " à sa place. Comme, ce faisant, elle a transformé la relation privilégiée qu'elle entretenait avec un génie en "héritage", les Sénoufo Tyebara affirment volontiers à propos du sandogi du matrilignage que «c'est l'amitié qui est devenu fardeau ".

Selon qu'ils sont envoyés par un génie ou par un ancêtre, les signes d'élection interprétés comme tels par les devins ne sont pas les mêmes. Ainsi qu'un ancêtre, un génie frappera son élue ou l'entourage de celle-ci de maladies et d'infortunes, mais contrairement à lui, il les fera précéder d'un de ces "événements insolites " (kanyon'mo) qui surviennent toujours en brousse et volontiers dans un contexte de travail. D'autre part, si l'ancêtre élit un individu en tant qu'il est un membre de son lignage, le génie, lui, élit un individu en tant qu'il est doté de qualités et de défauts - d'une personnalité propre. Le motif privilégié d'élection d'une personne par un génie est bien souvent un sentiment d'amitié : soit qu'elle affiche une beauté peu commune, soit

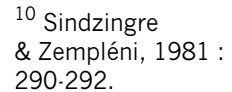


11 De même que l'amitié, l'inimitié ou le ressentiment peuvent également conduire un génie à élire un individu et à lui ordonner de porter son sandogi.

C'est alors une faute de comportement à son égard, telle la trans gression d'un interdit de travail ou le meurtre d'un animal qui lui est cher, qui justifie que l'instance « surprenne » la personne avant de lu ordonner de « prendre son travail » et de « porter » le sandogi. qu'elle excelle dans un domaine particulier, la personne élue par un génie pour "porter" son sandogi lui a plu. Le sentiment ressenti par le génie à l'endroit de l'individu auquel il se "colle" confine alors au sentiment amoureux. C'est toujours un génie du sexe opposé qui se " colle " à un individu; et parce qu'il le fait toujours en lui apparaissant continuellement dans des rêves, l'élue "perd l'envie " de tout autre que lui. Aussi entre-t-elle à tout propos en conflit avec son conjoint et n'enfante-t-elle pas ${ }^{11}$.

Mais par-delà les nombreuses différences qui singularisent leurs parcours respectifs, les deux sandobele (pl. de sandowi), élues pour l'une par un ancêtre et pour l'autre par un génie, ont en commun d'être tenaillées par un même sentiment de doute concernant leur élection: quand la souffrance infligée aux novices du pro était surtout physique, l'impétrante du sandogi expérimente une souffrance morale dont le sentiment de doute est l'expression dominante. Portant sur l'authenticité de son élection, ce sentiment l'envahit dès avant son initiation. Le tourment de la future initiée est d'ignorer si les devins n'ont pas été magiquement induits par quelque individu malveillant à lui dire de "porter" le sandogi. Or il est une chose dont elle peut être sûre : "porter » un sandogi qui n'était en réalité pas "pour sa tête " est la promesse d'une mort prochaine. C'est en effet à travers «les paroles du sandogi " qu'un ennemi " trouve à t'attraper " : "De la manière dont il invente un sandogi, si tu te trompes et que tu le portes, tu vas mourir. " Particulièrement vulnérables aux opérations magiques, les "paroles du sandogi » énoncées par les devins sont plus que toutes autres porteuses d'un doute mettant en cause la survie d'un individu.

Ce doute concernant son élection se nourrit du sentiment d'incrédulité que suscitent les implications de celleci. En effet, le doute de la future initiée est à la mesure de sa réticence à admettre son élection par un ancêtre ou un génie. Car l'élue n'est ni sûre ni heureuse de l'être. Bien loin de le réjouir, l'annonce faite à un individu de son élection suscite son désespoir, personne n'aspirant à la vie 
saturée de contraintes qui est celle de l'initiée au sandogi. Certes, les interdits auxquels elle doit se plier rappellent ceux du « jour de marché » ou « jour de repos » hebdomadaire imposé à tout individu par son double protecteur, le yigevowi. Ce jour venu, le yigevowi attend en tout premier lieu de sa "créature " qu'elle fasse sien son propre jour de repos et qu'elle s'abstienne de cultiver. Cet interdit de travail agricole s'accompagne également d'un ou de plusieurs interdits alimentaires, et de quelques prescriptions telles que celle de porter des insignes blancs. Mais les interdits prescrits à l'initiée au sandogi le sont par des puissances multiples et autrement plus exigeantes que le seul yigevowi. La sandowi est ainsi soumise à des interdits plus nombreux et, pour certains d'entre eux, permanents plutôt qu'hebdomadaires : la consommation de certaines nourritures et, plus grave, la pratique du travail agricole, lui sont interdites de façon définitive.

C'est en quelque sorte forte de ses doutes que la future initiée va tenter d'infléchir ce dont elle ne veut pas croire que ce soit ni son sort, ni la volonté d'une quelconque instance électrice. Bien loin de se satisfaire d'un diagnostic unique, celle qu'une première consultation divinatoire a promise à la vie contraignante et marginale de sandowi multiplie les visites chez différents devins avant de prendre en considération la possibilité de son élection. De nouvelles consultations divinatoires auront ensuite pour objet de négocier avec l'instance que la future initiée se sera sans doute résolue à considérer comme son instance électrice. J'ai déjà évoqué ailleurs les longs pourparlers que deux anciens champions de travail agricole, Zananiènè Soro et Pana Silué, avaient eus avec leurs génies respectifs. Car dans les rares cas où l'institution initiatique du sandogi recrute des membres masculins, il s'agit en général de cultivateurs d'exception. Il arrive parfois, en effet, qu'un jeune génie femelle, séduit par l'ardeur à la tâche de l'un d'eux, se " colle " à lui et exige qu'il «porte " le sandogi. Moins que tout autre prêt à interrompre son activité agricole, le champion met tout en œuvre pour éloigner de lui son encombrante "amie", avant d'être finalement contraint à entrer en pourparlers 
avec elle. Il se peut que ces négociations trouvent une issue favorable et que, comme Zananiènè Soro, il parvienne à satisfaire son instance électrice en faisant simplement sculpter une statuette la représentant, ou en faisant l'acquisition des différents objets qui constituent l'équipement de l'initié au sandogi. Mais il est plus probable que, comme Pana Silué, le cultivateur d'exception assailli par les infortunes que son génie lui envoie en cascade pour lui signifier avec insistance son souhait de le voir " porter » le sandogi, s'y résigne.

Mais pour le "porter" effectivement, il ne suffit pas que celui ou, plus souvent, celle dont c'est peut-être la destinée surmonte ses propres doutes; il faut encore que son entourage surmonte les siens. Nombreux sont tes cheminements vers l'initiation interrompus en raison des doutes nourris par les parents de celle qui a pourtant accepté son sort, telle Kodatcha. C'est au cours d'une longue maladie que Kodatcha a pour la première fois entendu de la bouche d'un devin qu'une ancêtre souhaitait la voir prendre son sandogi. Mais elle n'a pris ce diagnostic en considération que quelques années plus tard, lorsqu'il a été énoncé une seconde fois, peu de temps après le décès de son premier enfant. Elle n'a alors plus attendu avant de rassembler les icônes divinatoires avec lesquelles son ancêtre devinait et de cesser toute activité agricole. Mais ses oncles ne l'entendaient pas de cette oreille et ne perdaient pas une occasion de lui demander : "Tu ne cultives plus et tu manges. Est-ce que ce sont tes génies qui te nourrissent? "Seule sa mère lui apportait son soutien, lui procurant quotidiennement de quoi se nourrir et l'aidant à rassembler la somme d'argent et les biens nécessaires à son initiation prochaine. Mais le coût de l'initiation, trop élevé pour deux femmes isolées, eût raison de leur détermination. Kodatcha n'a jamais été initiée au sandogi. Plus d'une quinzaine d'années plus tard, elle le regrettait encore, y voyant la cause de ses fréquentes maladies comme de l'insuccès de ses champs d'arachide.

Dans d'autres cas, comme celui de Wandja, les réticences de l'entourage de l'élue ne font que retarder son initiation. Aussitôt après son mariage, Wandja avait mis au 
monde cinq enfants, tous morts avant même d'avoir fait leurs premiers pas. Les devins qu'elle consultait alors étaient unanimes : la mort de ses enfants était la manifestation du désir d'une ancêtre de la voir "prendre ses bagages ". À Wandja, " porter le sandogi " apparaissait comme un moindre mal en comparaison avec l'épreuve de voir ses enfants mourir et de ne pas être mère. Au plus profond d'elle-même, elle se disait : "Si c'est cela qui tue mes enfants, que je trouve un enfant. Si ce n'est pas cela qui les tue, que je le sache aussi. " Mais les oncles maternels de Wandja voyaient les choses autrement : une seule place d'initiée au sandogi du lignage était vacante, et selon eux, la dernière personne à l'avoir occupée en était morte. En effet, l'ancêtre qui demandait à Wandja de "prendre ses bagages " ne pouvait être que sa tante maternelle, décédée prématurément et sans avoir eu d'enfant aussitôt après avoir " habillé le sandogi ": "Le sandogi était sorti derrière elle. On a attaché le sandogi sur elle, elle l'a habillé et elle est morte. Personne ne l'a plus porté. Notre oncle a dit qu'elle était morte sans avoir mis d'enfant au monde, que personne n'allait plus porter son sandogi. " Wandja elle-même était parfaitement consciente que la "parole du sandogi " avait pu être mise dans la bouche des devins par un individu malintentionné à son égard. Mais quand Wandja elle-même était tombée malade, ses oncles avaient finalement accepté qu'elle "porte " le sandogi de sa tante et qu'elle soit initiée.

Il en va pour la plupart des individus auxquels les devins ont transmis le souhait d'une instance électrice de les voir "porter" le sandogi comme pour Wandja: leurs doutes et ceux de leur entourage ne font que différer le moment de l'initiation. Mais que celle-ci soit finalement programmée et organisée ne signifie toutefois pas que tous les doutes ont été levés. Un peu comme les futurs initiés du Bwiti gabonais, ce sont des êtres sceptiques qui sont initiés, et initiés en tant que tels, au sandogi ${ }^{12}$. De sorte que la future sandowi se trouve bientôt engagée dans un processus initiatique dont bon nombre d'épreuves visent précisément à s'assurer que ce sandogi était bien " pour sa tête ».

12 Bonhomme, 2005. 


\section{Le doute au cœur de l'initiation et au-delà}

La première des trois étapes que la novice est amenée à franchir s'étend sur une semaine au cours de la saison sèche, et est appelée « attacher le sandogi ». Le premier jour, la novice est rasée, revêtue d'un pagne blanc, et autour de ses hanches est attachée une fine cordelette appelée " corde de la souillure ». Ainsi apprêtée, elle "part à l'eau " ou "descend au marigot" en la seule compagnie de quelques sandobele. La même scène de "départ au marigot " est répétée chaque matin et chaque soir de cette première semaine d'initiation. À son terme, les parents de l'impétrante ne manqueront pas de l'observer attentivement en quête du principal indice témoignant de ce que le sandogi qu'elle s'apprête à "porter " n'était pas destiné à quelqu'un d'autre : l'impétrante doit avoir pris du poids. Il est préférable que son entourage soit en mesure de la trouver ainsi embellie, car il n'est plus temps de revenir en arrière, le sandogi étant d'ores et déjà "soulevé ", comme en témoigne «la poule du soulèvement du sandogi " que le mari de la novice, ou son père si elle n'est pas mariée, lui a remis à l'intention de l'association des sandobele. Après avoir reçu la poule, la responsable de l'initiation peut songer à couper la « corde de la souillure " pour la remplacer par une autre cordelette de la même couleur blanche. Il importe que celle-ci ne se rompe pas avant la prochaine étape de l'initiation qui a lieu six mois plus tard. Si tel était malgré tout le cas, le lignage de la novice pourrait se préparer à la survenue d'événements malheureux, parmi lesquels la mort de la novice elle-même, sans doute initiée à tort. Il reste qu'à l'issue de cette première étape initiatique, la novice est "sandowi propriétaire de cendre fraîche ", statut qu'elle conserve jusqu'à la fin de son initiation et qui s'accompagne de très nombreuses prescriptions et d'autant d'interdits. Quelques-uns de ces interdits et obligations sont néanmoins levés après que la novice a franchi l'étape médiane de son initiation : " raser la tête noire".

Cette étape médiane voit l'association villageoise des sandobele se réunir pour répéter quelques-uns des gestes déjà accomplis lors du rituel appelé "attacher le sandogi » : 
raser la tête de la novice, couper l'ancienne cordelette et en attacher une nouvelle sur ses hanches. Mais cette étape fait également intervenir des éléments nouveaux, et parmi eux, le don fait par la responsable de l'initiation à la novice du bracelet en forme de python, des icônes divinatoires et de la calebasse-hochet qui constituent les principaux attributs d'une sandowi. La novice s'empresse alors de les déposer dans la petite "maison de sandowi " que l'un de ses parents aura construite pour elle en prévision de cette étape de son initiation. En outre, l'étape "raser la tête noire " est autrement plus festive que celle dénommée " attacher le sandogi » : réunies quatre jours durant, les sandobele chantent, dansent, cuisinent puis consomment les ignames, le miel et les poulets que le père (ou le mari) et l'oncle de la novice ont rassemblés pour les leur donner. Cette humeur festive n'est pas sans lien avec l'heureuse rupture que marque cette étape. Car à la suite de l'étape "raser la tête noire " et jusqu'à l'étape finale de son initiation, la novice est affranchie de la plupart de ses obligations et interdits, et tout particulièrement de son interdit de travail agricole. Autorisée à cultiver par ses génies, la novice peut ainsi espérer réunir d'elle-même et plus rapidement la quantité de nourriture et la somme considérable d'argent qu'exige l'association des sandobele pour clore l'initiation. Mais en dépit de cet assouplissement certain des contraintes pesant sur la novice, la tension reste grande : la plus haute attention est ainsi portée, par elle comme par son entourage proche, à son aspect extérieur comme précédemment, mais également à ses rêves. Nombreux sont en effet les rêves qui, parce qu'ils suggèrent qu'elle n'aurait pas dû être initiée, appellent une série de consultations divinatoires. Rêver qu'elle franchit un interdit tel celui de cultiver ou que la cordelette attachée autour de ses reins s'est rompue, est du plus mauvais augure pour une novice en voie d' " habiller " le sandogi.

Dernière étape de l'initiation, "habiller le sandogi" dure une semaine et se déroule au cours de la saison pluvieuse. La novice est, avant toute chose, rasée une dernière fois. Elle est ensuite conduite en brousse où il lui est ordonné de "prendre le trriwi ", un objet connu des seules 
sandobele. Le temps qu'il faut à la novice pour trouver l'objet préalablement dissimulé est inversement proportionnel au temps qu'il lui reste à vivre. Il se peut que la novice le cherche en vain : le sandogi qu'elle a pourtant d'ores et déjà attaché n'était donc en réalité pas "le sien ", et il la tuera sans doute prochainement. Aussi les sandobele ne rentrent-elles jamais de brousse sans un chant à la bouche dans lequel elles affirment, comme pour s'en convaincre, que "notre enfant sandowi va vieillir, notre enfant sandowi va vivre". Après s'être une dernière fois lavée au marigot, la novice enduit son corps d'une épaisse couche de beurre de karité : si la cendre fraîche manipulée tout au long de l'initiation permettait de la "rafraîchir », le beurre de karité ne permet rien de moins que d'"éteindre " la novice sur laquelle toutes les "chaleurs " et les « souillures " du lignage se sont concentrées. "Sandowi propriétaire de cendre fraîche " jusqu'alors, la novice est désormais simplement sandowi. Une fois "éteinte ", elle est revêtue d'un joli pagne, parée d'un foulard pareillement coloré, et ornée d'une multitude de bijoux. Car c'est une grande fête à laquelle s'apprêtent les sandobele, au cours de laquelle elles chantent, dansent et font bombance : la novice et ses parents leur ont remis une vingtaine de poulets, une douzaine d'ignames, du miel, et trois paniers remplis l'un de riz, l'autre de maïs et le troisième de mil.

La souffrance initiatique des novices du sandogi n'est donc pas celle, principalement physique, des novices du pro. Certes, quelques-uns de leurs interdits, tel celui de se laver avec de l'eau froide, sont présentés par les Sénoufo comme une source de souffrance physique; mais cet accent mis sur leur souffrance physique, comme celui mis sur la souffrance morale des novices du pros, semble plutôt dériver de la conception sénoufo de la souffrance : une souffrance nécessairement entière, dont les deux dimensions, physique et morale, viennent en renfort l'une de l'autre ${ }^{13}$. L'expérience de la future sandowi, avant et pendant son initiation, est avant tout de l'ordre du tourment. Tandis que son corps est d'autant plus choyé que sa beauté est le 
meilleur indice de ce que son sandogi était bien "le sien ", son esprit, lui, est sans cesse inquiété. Seule, elle poursuit un parcours dont il faut qu'elle se demande à tout instant si, faute d'être sa véritable destinée, il ne la conduit pas à la mort.

Ce questionnement ne prend d'ailleurs pas fin avec l'initiation. Au-delà, le doute persiste éventuellement à travers la pratique de la divination que leur génie prescrit à la plupart des sandobele. Car c'est au sein d'une institution initiatique où le doute occupe une place centrale que sont recrutés ceux qui doutent de dire la vérité à leurs clients. Le devin souffre continuellement de ne pas savoir si, en transmettant les paroles que des génies lui ont soufflées, il transmet des paroles empreintes de vérité ${ }^{14}$. Pourtant, la sandowi ne peut que se réjouir de ce que son génie l'ait destinée à la divination. Ce faisant, il lui a " ouvert son intérieur " et proposé une activité qui, si elle n'est pas aussi valorisée ni valorisante que l'activité agricole, a le mérite d'en être une. Il reste que la vie des sandobele, qu'elles pratiquent la divination ou non, est une perpétuelle remise en cause, par ellesmêmes ou par leur entourage, de leur statut de sandowi. Le prolongement du parcours biographique de Wandja Soro en témoigne.

Peu de temps après avoir « habillé le sandogi », Wandja avait mis au monde un petit garçon auquel, pleine d'espoir, elle avait donné le prénom évocateur de Tchalanion, "Bonne divination». Mais à la suite de ses oncles, la mère de Wandja, Piéréguénion, avait commencé à émettre des doutes quant à "l'authenticité " du sandogi que portait sa fille. L'installation dans le village d'un fétiche qui interdisait nombre d'objets de culte et ordonnait à leur possesseur de les " jeter", raffermit encore les doutes de Piéréguénion. Pas un jour ne passait sans qu'elle houspille sa fille : «Ma mère disait que je n'aimais pas le travail, que les autres [sandobele] avaient jeté leur sandogi et étaient reparties cultiver. C'est donc sa petite sœur [la petite sœur de l'ancêtre dont Wandja avait porté le sandogi : la mère de Wandja] qui m'insultait et me disait que j'étais paresseuse, sans 
${ }^{15}$ Me conter son histoire permettait sans doute à Wandja de rappeler à sa mère ses erreurs de jugement : assise non loin de nous et appa. remment absorbée par la préparation du dîner, Piéréguénion ne pouvait pas ne pas entendre les propos critiques de sa fille à son égard. quoi j'allais jeter mon sandogi. Elle me disait: "Quel bien te fait-il?" "Wandja s'était alors tournée vers son oncle, qui lui avait confirmé que "tous les sandoyi (pl. de sandogi) ne peuvent pas être jetés». Pourtant, Wandja n'avait pas résisté à la pression que sa mère faisait quotidiennement peser sur elle: elle avait finalement "jeté " son sandogi et repris sa houe. Elle n'avait pas tardé à en éprouver du regret. Son fils Tchalanion était tombé malade - maladie que les devins interprétèrent aussitôt comme la manifestation du courroux de l'ancêtre dont le sandogi avait été jeté. Wandja s'était alors rendue auprès de l'autel du sandogi du lignage pour y prononcer ces paroles : "Je suis allée dire à la défunte que ce n'est pas moi qui voulait le jeter, que c'est sa parente qui voulait que je le jette. Je lui ai dit de ne pas me tourmenter, de ne pas tourmenter mon enfant, je lui ai dit de lutter avec sa parente. "Et en effet, Piéréguénion ${ }^{15}$ était tombée gravement malade à son tour, ce qui provoqua aussitôt cette réaction de la part de Wandja : "J'ai dit que si elle n'était pas morte avant le jour de kakpogi, j'allais croire que c'était le sandogi et j'allais le reprendre. J'ai dit que si elle mourrait avant ce jour, je n'allais plus jamais le porter, je n'allais plus jamais le prendre. Quand ce jour est venu, je suis allée chez elle et je l'ai appelée plusieurs fois. Je l'ai entendue répondre tout doucement : "mm." Je lui ai alors dit: "N'est-ce pas toi qui m'as dit d'aller jeter le sandogi de ta grande sour? On dit que c'est ce qui te rend malade, et c'est ce que je vais aller reprendre." Elle a dit : "Oui." On a dit : "Elle parle !" Je lui ai encore dit : "C'est toi qui m'avais dit d'aller le jeter. Tu m'as dit de ne pas le porter, et mes enfants sont morts. Je t'ai suppliée et je l'ai porté alors que j'étais déjà âgée. Quand je me suis débrouillée pour mettre au monde celui-ci, tu m'as encore dit de le jeter. Mais mon enfant ne mourra pas. C'est sur toi que cela va rester, et non sur mon enfant. Je l'ai bien dit à ta parente. Et maintenant, je vais le prendre. Est-ce que je vais le prendre?" Elle a dit : "Oui, va le prendre." Et tout le monde a cru, et j'ai repris les bagages. "Non seulement sa mère et son enfant ont guéri, mais encore Wandja a mis au monde un second garçon en aussi bonne santé que son frère aîné. 


\section{Initiation et élection}

Comment comprendre ce qui peut finalement apparaitre comme un méticuleux entretien du doute plutôt que comme un laborieux cheminement vers une certitude? Le doute semble en premier lieu etre la marque distinctive d'une initiation qui recrute ses membres par voie d'élection. Bien loin d'être le pendant de l'initiation obligatoire et masculine au pro, le sandogi est l'une de ces initiations électives qui pourraient bien se caractériser par la place que le doute y occupe nécessairement. «Suis-je bien élu?» est en effet la question qui taraude les novices du sandogi, et eux seulement. Aussi malmenés qu'ils soient, les impétrants du poro ne peuvent douter d'être à leur place au cours d'une initiation imposée à l'ensemble des hommes. Bien au contraire, leur initiation leur apporte, de la même manière qu'aux novices des initiations donnant accès au statut d'adulte de plein droit chez les Gbaya Kara, la sécurité : «sécurité sous forme de réconfort dès le moment de l'initiation par la révélation qui leur était faite à cette occasion du rôle important qu'ils devaient jouer dans la société. L'adolescent et sa compagne n'avaient pas à se poser la question de savoir pourquoi ils étaient là, pourquoi ils existaient et quelle fonction était la leur $[. . .]^{16}$.»

Et cette question que la novice du sandogi est seule à se poser, elle se la pose dans la solitude. Que l'initiation élective au sandogi soit individuelle et non pas collective comme l'initiation obligatoire au pros semble être pour beaucoup dans le fait que le doute y prend le pas sur la douleur. Contrairement aux novices réunis en un collectif, c'est dans la plus grande solitude que la novice du sandogi franchit les étapes d'une initiation dont elle n'est pas absolument certaine que les franchir soit effectivement ni son destin ni la volonté d'une quelconque instance électrice. On retrouve ailleurs cette distribution d'une souffrance physique et d'une souffrance plus intérieure entre les initiations collective et individuelle : chez les Béti du Cameroun ${ }^{17}$, la composante physique de la souffrance diminue pareillement à mesure que l'initiation est plus individuelle. Très marquée

\footnotetext{
${ }^{16}$ Vidal, $1976: 291$

${ }^{17}$ Mallart Guimera, 1989.
} 
dans l'initiation « tribale » obligatoire, elle est, sinon moins présente, moins arbitraire dans l'initiation au culte des ancêtres, et disparaît presque totalement dans l'initiation des grands thérapeutes et des joueurs de harpe-cithare. Ces derniers ne subissent ni les épreuves douloureuses et humiliantes de l'initiation obligatoire et collective, ni les épreuves douloureuses et purificatrices du culte des ancêtres; ce qu'ils doivent surmonter est avant tout la peur de faire face à un monde inconnu tout au long d'un parcours périlleux.

Et cette solitude des novices du sandogi se maintient encore après leur initiation et continue de renforcer l'opposition entre les initiations individuelles et les initiations collectives. Car quand l'initiation au pro fait accéder un collectif de jeunes garçons au statut d'adulte de plein droit, leur permettant par là d'intégrer un groupe majoritaire, l'initiation élective et individuelle du sandogi permet tout au plus à un individu d'intégrer un groupe marginal. De sorte que pour paraphraser Pascal Boyer ${ }^{18}$ qui établissait une relation nécessaire entre la construction, métaphorique ou métonymique, des énoncés initiatiques et le status des énonciateurs, on peut dire qu'il existe une relation nécessaire entre la construction de leur souffrance, morale ou physique, et le status des initiés. De même en effet qu'une langue secrète fondée sur des manipulations métaphoriques plutôt que métonymiques parait tout indiquée pour des novices destinés à intégrer un groupe déviant, de même le sentiment de doute plutôt que la souffrance physique paraît tout indiqué pour animer des novices qui seront très prochainement dans la position forcément inconfortable d'initiées au sandogi et, pour un certain nombre d'entre elles, dans la position à peine plus enviable de devins.

Aussi la profonde réticence des élues à "porter " le sandogi se comprend-elle sans peine. Et si l'initiation élective au sandogi s'oppose aux initiations faisant accéder au statut d'adulte de plein droit, elle s'oppose tout autant aux initiations facultatives et volontaires appelées par une vocation. Un peu comme le calviniste dépeint par Weber, la future initiée au sandowi doute d'être élue, mais contrairement à lui, elle n'aspire pas à l'être. Aussi le doute lui 
apparait-il dans un premier temps comme ce qui lui permettra peut-être d'échapper à la destinée si peu enviable de sandowi. Mais l'initiation au sandogi est dans le même temps la solution qui lui est proposée pour mettre fin à une succession d'infortunes, et même, du fait de la dimension de doute qui s'y maintient, la solution la plus confortable. Car l'intranquillité à laquelle le doute condamne la sandowi n'est pas sans rappeler la "douce inquiétude " en laquelle Jean Deprun nous dit que Leibniz, et d'autres philosophes du XviII e siècle avec lui, voyait "la condition de la félicité " plutôt que "son négatif ", une "fin " plutôt qu'un "obstacle $^{19}$ ». Peut-être en effet le doute est-il un fardeau pour l'initiée au sandogi, mais il n'est pas que cela; il est aussi ce qui lui octroie une mobilité salvatrice. Car quand l'initiation des garçons au pro leur offre une condition nouvelle et définitive, l'initiation d'une fille au sandogi lui offre au contraire, au gré des événements, heureux ou malheureux, qui surviennent dans sa vie, la latitude de se repositionner par rapport à l'institution initiatique du sandogi, à quelques instances supra-humaines et aux membres de son entourage. Le doute rend ainsi le sandogi à même de secourir ses membres en leur proposant une condition qui porte en elle une exigence de perpétuelle réévaluation. Et c'est finalement une forme de quiétude qui peut découler de ce doute assumé, ainsi que l'était celui des sceptiques grecs pour qui " la cause principale du scepticisme " résidait dans "l'espoir de connaître la quiétude ${ }^{20} »$.

\section{Initiations féminine et masculine}

Ainsi, la présence du doute au sein de l'initiation au sandogi tient en partie à ce qu'elle est une initiation élective. Mais elle tient aussi et peut-être surtout à ce qu'elle est une initiation féminine. Certes, quelques hommes sont initiés au sandogi et les raisons si particulières pour lesquelles ils le sont m'ont souvent conduite à leur accorder toute mon attention ${ }^{21}$. Mais ces hommes constituent des exceptions au sein d'une institution initiatique très fortement féminisée $e^{22}$. Or la prédominance de la souffrance morale sur la

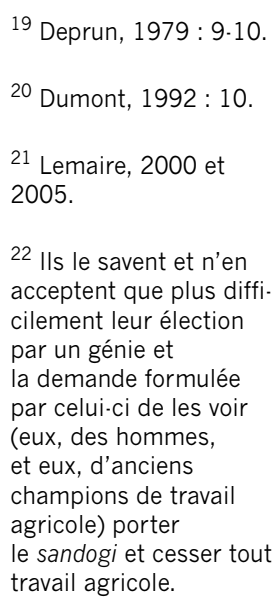


23 Vidal, 1976 : 227.

${ }^{24}$ Richards, 1992 [1956]. souffrance physique qui caractérise l'initiation au sandogi se retrouve dans de nombreuses autres initiations féminines. Et dans ces dernières, la souffrance morale se colore également volontiers de la dimension de doute, de tension, d'inquiétude. Non pas que les initiations féminines ne comprennent jamais d'épreuves physiquement pénibles; elles en comprennent parfois de très douloureuses, mais celles-ci semblent être volontiers passées sous silence, à l'inverse des épreuves douloureuses dont les initiés masculins aiment souvent à rappeler qu'ils les ont surmontées. C'est ainsi que chez les Gbaya Kara, les initiées taisent toute leur vie durant la souffrance associée à la clitoridectomie qu'elle ont subie dans le cadre du bana ${ }^{23}$.

En revanche, les souffrances morales endurées lors des initiations féminines ne s'entourent pas de la même discrétion. Certaines initiations féminines, tel le chisungu des jeunes filles bemba décrit par Audrey Richards ${ }^{24}$, se présentent même comme une succession d'épreuves morales empreintes d'une dimension de doute. Le chisungu s'ouvre en effet sur des moqueries à l'adresse des novices - moqueries qui resurgiront régulièrement tout au long de l'initiation, et dont l'assistance attend avec une certaine anxiété qu'elles suscitent leurs pleurs. L'initiation se poursuit avec des épreuves qui n'en seraient pas si l'assistance n'exagérait pas tant leur difficulté ni n'insistait tant sur la nécessité, pour les novices, de les surmonter. Mais le doute introduit par l'assistance et le climat de tension qui en découle en font des épreuves véritables, moralement douloureuses, que toutes les novices ne surmontent pas. C'est ainsi avec la plus grande appréhension que l'assistance scrute la novice et se demande si, oui ou non, elle va réussir à sauter par-dessus un modeste monceau de branchages. Et c'est au soulagement de tous qu'elle finit par y parvenir. D'autres épreuves du chisungu, plus ou moins difficiles, s'accompagnent pareillement d'une interrogation quant à leur réussite par les novices : attraper de petits insectes avec sa bouche, allumer un nouveau feu, mettre à mort des poulets. Autant de moments où semble se jouer le succès d'une initiation féminine et où le doute prend une place équivalente à celle occupée par la douleur dans les initiations masculines. 
Nombreuses sont d'ailleurs les initiations féminines où, comme pour le sandogi, se retrouve un trait qui caractérisait déjà les initiations électives et y introduisait le doute : les initiations féminines, ou tout au moins celles qui conditionnent le passage au statut d'adulte de plein droit, sont volontiers solitaires. Plus que les jeunes garçons en effet, "individual transitions to maturity usually concern girls and are closely linked to events in the life cycle, such as the development of the breasts or the onset of menstruation $[\ldots]^{25}$ ". Certaines de ces initiations féminines qui donnent accès au statut d'adulte sont malgré tout collectives : la dimension de solitude est alors comme préservée à travers le sort réservé à l'une des novices. Il n'est pas rare en effet que les initiations féminines prévoient d'extraire une "première " du collectif des impétrantes. Tel est le cas du dikuntri, l'initiation féminine des Tamberma du Togo ${ }^{26}$ : de la « grappe compacte et anxieuse " des novices tamberma se détache "la jeune fille calme comme la surface du marigot", une "première " choisie pour sa grâce physique et la douceur de son caractère. En dépit du prestige qui ne manque pas de lui être associé, aucune jeune fille tamberma n'aspire à ce statut de "première ». Car il l'expose à la force mortifère et stérilisante de la puissance fakuntifa sur laquelle repose l'initiation. Une telle exposition la destine à une vie scandée par de multiples interdits et à une mort précoce. Il est probable également qu'elle l'empêche d'avoir des enfants en nombre suffisant, à moins qu'elle ne la rende tout à fait stérile. Aussi le choix de la "première " est-il, pour les membres du clan au sein duquel elle est élue, à la source d'âpres négociations menées dans une atmosphère tendue. Quant à la «première " elle-même, elle n'apprend pas la nouvelle de son élection sans en éprouver une profonde angoisse qui l'incite parfois à fuir.

Certes, quelques initiations masculines distinguent elles aussi un individu du collectif des impétrants et lui réservent un sort un peu différent. Chez les Gbaya Kara ${ }^{27}$, un chef de groupe est désigné dans l'initiation masculine du labi comme dans l'initiation féminine du bana. Mais il ne semble pas que ninan, le chef de groupe des postulants mas-

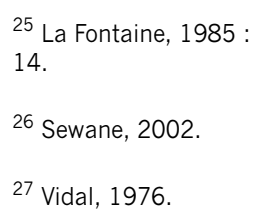

27 Vidal, 1976 
culins, vive en tant que tel une expérience aussi distincte de celle des autres novices que ndonem, son homologue féminin, ni aussi moralement éprouvante. Plutôt que sur ninan lui-même, c'est sur son père que semble peser toute la responsabilité de l'initiation. Que celle-ci tourne mal, et le malheur s'abattra sur celui qui, par le geste de désigner son fils comme chef de groupe, l'a déclenchée et prise en charge. Il en va un peu différemment pour ndonem, qu'un épisode de l'initiation féminine met dans une position aussi inconfortable que centrale. Au cours de leur temps de retraite dans le camp initiatique, l'esprit du bana se manifeste aux impétrantes à travers une étape initiatique d'une grande violence émotionnelle. Par une nuit sans lune, alors qu'elles sont pour la plupart assoupies, des sons inquiétants parviennent à leurs oreilles. Quelques instants plus tard, une partie du toit de leur case s'affaisse et à travers des caresses et des coups de griffe, elles subissent bientôt le contact humide d'un être mystérieux. Mais si l'heure est à l'épouvante pour l'ensemble des novices, elle l'est à un degré encore supérieur pour l'une d'entre elles: ndonem est en effet soulevée de terre puis enlevée par la ténébreuse puissance de cet épisode initiatique. Encouragées par leurs parents arrivés sur les lieux à rechercher leur compagne, les novices ne la retrouveront que plusieurs heures plus tard, dans un état et une position qui leur donnent à penser que moko, l'esprit du bana, l'a déflorée.

En réalité, la jeune fille enlevée par moko peut être une autre que ndomen. Mais l'identité de la novice isolée par cette épreuve individuelle importe peu. L'essentiel est que cette initiation féminine, comme beaucoup d'autres, réserve à l'une de ses novices une expérience particulière et particulièrement éprouvante d'un point de vue émotionnel, et que les autres novices en soient les témoins. Tout se passe en effet comme si l'expérience exemplaire d'une seule rejaillissait sur le parcours initiatique de chacune et lui donnait la dimension de solitude indispensable à la bonne réception et au renforcement de la souffrance morale qui, dans les initiations féminines, semble plus que tout autre élément à l'œuvre dans la transformation des novices. De 
fait, Pierre Vidal voit dans cette épreuve initiatique de la manifestation de l'esprit du bana un moment déterminant dans la métamorphose de «fillettes apeurées de l'intronisation » en « jeunes filles prêtes à être femmes, moqueuses et sûres d'elles-mêmes ».

Ne se pourrait-il d'ailleurs pas que le type de transformation visé respectivement par l'initiation masculine et l'initiation féminine explique le recours, pour l'une à la souffrance physique, pour l'autre à la souffrance morale? La souffrance physique de l'initiation masculine apparait en effet comme le moyen d'instaurer une complète métamorphose; elle est, pour reprendre le mot et les analyses de Michael Houseman" ${ }^{28}$, le "mal pour le mâle " que le novice ne peut devenir qu'au prix d'une rupture radicale avec sa masculinité naturelle. Rien de tel en ce qui concerne la souffrance de l'initiation féminine au sandogi : sous la forme du doute, elle est présente pendant l'initiation, mais aussi en amont et en aval de celle-ci. Le doute y est tout à la fois un préalable et un aboutissement, et encore n'y est-il qu'une forme exacerbée d'un sentiment que, en tant que femmes, les novices avaient d'ores et déjà amplement éprouvé avant même d'être pressenties comme de probables initiées. Dans le cas sénoufo en effet, la prédominance de la souffrance morale sur la souffrance physique au sein de l'initiation au sandogi apparaît en réalité comme le prolongement d'une expérience féminine plus étendue. Les Sénoufo distribuent volontiers les souffrances physiques et morales entre les sexes, attribuant la souffrance physique aux hommes, et la souffrance morale aux femmes. C'est ainsi que les chants funéraires entonnés par les femmes à la mort de leur parent ou ami du sexe opposé retracent la souffrance physique que lui ont procurée ses travaux agricoles. Ils célèbrent en l'homme défunt le cultivateur qui a souffert physiquement en tant que tel, mais a désormais achevé son travail et peut enfin "trouver la respiration", autrement dit se reposer. Que le défunt soit une femme, et il en va tout autrement : si les chants funéraires évoquent pareillement le travail qu'elle a accompli durant sa vie, la souffrance qu'elle a endurée à travers lui, la fatigue qu'il lui a procurée et le repos que

28 Houseman, 1984 et 1986 
la mort lui donne enfin, le travail de la défunte dont les chanteuses retracent le douloureux parcours n'est pas celui, agricole, qu'elle a pourtant réalisé avec la même ardeur que son mari, sa souffrance n'est pas celle, physique, ressentie après l'avoir tout aussi ardemment réalisé, et son repos n'est pas celui de la cultivatrice. Le travail, la souffrance et la fatigue de la défunte sont autres. Plus que fatigue, ils sont tourments. Tourment d'avoir, en vertu de la règle d'alliance, été séparée de ses parents et pressurée par ses beauxparents - tourment qui ne cesse qu'à sa mort, à la suite du rituel qui lui permet de réintégrer enfin son matrilignage; tourment aussi, plus que fatigue de les avoir portés et élevés, d'avoir mis au monde des enfants et de les avoir vus souffrir de maladies et peut-être mourir.

Au lieu de permettre une métamorphose comme l'initiation masculine, l'initiation féminine autorise ainsi, à l'aide du sentiment de doute, une transformation sans discontinuité consistant en une intensification d'une identification sexuelle préexistante. Tout se passe en effet comme si les femmes étaient d'emblée ce qu'elles étaient destinées à être, et comme si l'initiation de quelques-unes d'entre elles ne leur apportait que de l'être avec plus d'intensité, faisant d'un petit nombre d'élues autant de figures ultimes de la féminité. Pour être inconfortable, le statut n'en est pas moins opportun. Car contrairement encore à celui conféré par l'initiation obligatoire et collective des garçons au poro, celui conféré par l'initiation élective et solitaire des femmes au sandogi, constamment mis en doute, n'est pas définitif. Et si douter d'être à sa place est douloureux, douter d'être à sa place permet aussi d'en changer. 


\section{Références bibliographiques}

Bochet, G.

1959 "Le poro des diéli », Bulletin de l'IFAN XXI (1-2), série B, pp. 61-102.

1993 "Le poro des Sénoufo ", in Arts de la Côte d'Ivoire, Paris, Musée BarbierMueller, pp. 54-85.

Bonhomme, J.

2005 Le Miroir et le crâne. Parcours initiatique du Bwete misoko (Gabon), Paris, CNRS Éditions.

Boyer, P.

1980 "Les figures du savoir initiatique ", Journal des Africanistes 50 (2), pp. 31 57.

Deprun, J.

1979 La Philosophie de l'inquiétude en France au XVIII siècle, Paris, Vrin.

Dumont, J.-P. (dir.)

1992 Les Sceptiques grecs, Paris, PUF.

Houseman, M.

1984 "Les artifices de la logique initiatique », Journal des Africanistes 54 (1), pp. 41-66.

1986 "Le mal pour le mâle : un bien initiatique ", in J. Hainard \& R. Kaehr (dir.), Le Mal et la douleur, Neuchâtel, Musée d'ethnographie, pp. 89-99.

La Fontaine, J. S.

1985 Initiation, New York, Penguin Books.

Lemaire, $\mathrm{M}$.

2000 Les Représentations du travail en pays sénoufo tyebara (Côte d'Ivoire), thèse de Doctorat, Paris X.

2005 "Le travail de la souffrance. Parcours biographiques du cultivateur sénoufo (Côte d'Ivoire) ", Systèmes de pensée en Afrique noire 17, pp. 71-90.

Mallart Guimera, L.

1989 "Initiations collectives, initiations individuelles", in Singularités. Textes pour Eric de Dampierre, Paris, Plon, pp. 291-312.

Richards, A.

1992 [1956] Chisungu. A girl's initiation ceremony among the Bemba of Zambia, Londres, Routledge.

Sewane, D.

2002 La Nuit des Grands Morts. L'initiée et l'épouse chez les Tamberma du Togo, Paris, Economica.

Sindzingre, N. \& A. Zempléni

1981 "Modèles et pragmatique, activation et répétition : réflexions sur la causalité de la maladie chez les Sénoufo de Côte d'Ivoire ", Social Science and Medecine XV (3), pp. 279-293. 
Vidal, P.

1976 Garçons et filles. Le passage à l'âge d'homme chez les Gbaya Kara, Nanterre, Labethno.

Zempléni, A.

1993 "L'invisible et le dissimulé. Du statut religieux des entités initiatiques ", Gradhiva 14, pp. 3-14.

1996 «Savoir taire. Du secret et de l'intrusion ethnologique dans la vie des autres ", Gradhiva 20, pp. 23-41. 